\title{
An exploratory, randomised, placebo-controlled, 14 day trial of the soluble guanylate cyclase stimulator praliciguat in participants with type 2 diabetes and hypertension
}

\author{
John P. Hanrahan ${ }^{1}$ (D) - Jelena P. Seferovic ${ }^{1}$ - James D. Wakefield ${ }^{1}$ • Phebe J. Wilson ${ }^{1}$ • Jennifer G. Chickering ${ }^{1}$. \\ Joon Jung ${ }^{1} \cdot$ Kenneth E. Carlson ${ }^{1}$ • Daniel P. Zimmer ${ }^{1}$ - Andrew L. Frelinger $\mathrm{III}^{2} \cdot$ Alan D. Michelson ${ }^{2} \cdot$ Linda Morrow $^{3}$. \\ Michael Hall $^{4} \cdot$ Mark G. Currie $^{1} \cdot$ G. Todd Milne ${ }^{1} \cdot$ Albert T. Profy $^{1}$
}

Received: 7 August 2019 / Accepted: 31 October 2019 /Published online: 19 December 2019

(C) The Author(s) 2019

\begin{abstract}
Aims/hypothesis Praliciguat (IW-1973), a soluble guanylate cyclase stimulator, amplifies nitric oxide signalling. This exploratory trial investigated the safety, tolerability, pharmacokinetic profile and pharmacodynamic effects of praliciguat in individuals with type 2 diabetes and hypertension.

Methods This Phase IIA, double-blind, placebo-controlled trial investigated praliciguat in 26 participants with type 2 diabetes and hypertension on stable glucose- and BP-lowering therapies. Participants were randomly allocated in a 3:5:5 ratio to three groups: placebo $(n=6)$, praliciguat $40 \mathrm{mg}$ once daily for days $1-14(n=10)$, or praliciguat $20 \mathrm{mg}$ twice daily for days $1-7$ then $40 \mathrm{mg}$ once daily for days 8-14 $(n=10)$. Assessments were made in clinic and included treatment-emergent adverse events, pharmacokinetics, metabolic variables, $24 \mathrm{~h}$ BP and heart rate, platelet function, reactive hyperaemia index (RHI) and plasma biomarkers. Participants, the sponsor, the investigator and clinic study staff (except designated pharmacy personnel) were blinded to group assignment.

Results Participants treated for 14 days with praliciguat had least-square mean change-from-baseline differences vs placebo $(95 \% \mathrm{CI})$ of $-0.7(-1.8,0.4) \mathrm{mmol} / \mathrm{l}$ for fasting plasma glucose, $-0.7(-1.1,-0.2) \mathrm{mmol} / \mathrm{l}$ for total cholesterol, $-0.5(-1.0,-0.1)$ $\mathrm{mmol} / \mathrm{l}$ for LDL-cholesterol, $-23(-56,9)$ for HOMA-IR in those not being treated with insulin, and $-5(-10,1) \mathrm{mmHg}$ and 3 $(-1,6)$ beats/min for average $24 \mathrm{~h}$ mean arterial pressure and heart rate, respectively. Apart from one serious adverse event (SAE; upper gastrointestinal haemorrhage), praliciguat was well tolerated. Praliciguat did not affect platelet function or RHI. Among exploratory biomarkers, plasma levels of asymmetric dimethylarginine decreased in praliciguat vs placebo recipients.

Conclusions/interpretation In participants with type 2 diabetes and hypertension on standard therapies, over 14 days praliciguat was well tolerated, except for a single SAE, and showed positive trends in metabolic and BP variables. These results support further clinical investigation of praliciguat.

Trial registration ClinicalTrials.gov NCT03091920.

Funding This trial was funded by Cyclerion Therapeutics.
\end{abstract}

Keywords BP · Cyclic guanosine monophosphate $\cdot$ Endothelial function · Hyperlipidaemia · Hypertension · Insulin resistance · Soluble guanylate cyclase stimulator · Type 2 diabetes

Electronic supplementary material The online version of this article (https://doi.org/10.1007/s00125-019-05062-x) contains peer-reviewed but unedited supplementary material, which is available to authorised users.

John P. Hanrahan

JHanrahan@Cyclerion.com

Cyclerion Therapeutics, Inc., 301 Binney Street, Cambridge, MA 02142, USA
2 Center for Platelet Research Studies, Dana-Farber/Boston Children's Cancer and Blood Disorders Center, Boston, MA, USA

3 ProSciento, Inc., Chula Vista, CA, USA

4 Ironwood Pharmaceuticals, Inc., Cambridge, MA, USA 


\section{Research in context}

\section{What is already known about this subject?}

- Soluble guanylate cyclase $(\mathrm{sGC})$ is a widely expressed signalling enzyme that catalyses the formation of cyclic GMP in response to NO binding

- $\mathrm{sGC}$ stimulators have been demonstrated to lower BP in animal and human studies

- $\quad \mathrm{sGC}$ stimulators have shown metabolic effects in animal models but effects on glucose and lipid metabolism have not been reported in humans

\section{What is the key question?}

- What is the safety, tolerability, and pharmacokinetic and pharmacodynamic profile of the sGC stimulator praliciguat when administered orally for 14 days to individuals with type 2 diabetes and hypertension?

\section{What are the new findings?}

- Praliciguat was generally well tolerated among participants with type 2 diabetes and hypertension on standard-ofcare therapy treated for 14 days

- Point estimates and associated $95 \% \mathrm{Cls}$ for changes from baseline suggested reductions in BP, fasting glucose, insulin resistance and serum lipid levels for participants treated with praliciguat

How might this impact on clinical practice in the foreseeable future?

- These results suggest that praliciguat warrants further investigation for the possible treatment of individuals with type 2 diabetes and its complications

\section{Abbreviations}

ABPM Ambulatory BP monitoring

ACEi ACE inhibitor

ADMA Asymmetric dimethylarginine

$\mathrm{AI}_{75} \quad$ Augmentation index corrected to a heart rate of 75 bpm

ARB Angiotensin receptor blocking agent

ARU Aspirin reaction units

bpm Beats/min

cGMP Cyclic GMP

$\mathrm{C}_{\max } \quad$ Maximum observed plasma concentration

DBP Diastolic BP

LS Least squares

MAP Mean arterial pressure

PPAR Peroxisome proliferator-activated receptor

PRU P2 $\mathrm{Y}_{12}$ reaction units

RHI Reactive hyperaemia index

SAE Serious adverse event

SBP Systolic BP

sGC Soluble guanylate cyclase

TEAE Treatment-emergent adverse event

$\mathrm{T}_{\max } \quad$ Time of $\mathrm{C}_{\max }$

TRAP Thrombin receptor activating protein

$\mathrm{V}_{\mathrm{Z}} / \mathrm{F} \quad$ Apparent volume of distribution during the terminal elimination phase

\section{Introduction}

The global prevalence of type 2 diabetes has increased rapidly over recent decades. In 2015, the Centers for Disease Control and Prevention reported that diabetes affected $9.4 \%$ of the US population, 90-95\% of whom had type 2 diabetes, and was the seventh leading cause of death [1]. Type 2 diabetes and its complications represent a substantial burden and cost to patients, providers, the healthcare system and society at large in the developed world.

Type 2 diabetes is associated with endothelial dysfunction, reduced NO bioavailability and impaired NO signalling [2, 3]. Endothelial dysfunction and disrupted NO signalling have been implicated in the pathophysiology of macro- and microvascular complications of diabetes [4]. Soluble guanylate cyclase (sGC), a central enzyme in the NO signalling pathway, catalyses the conversion of GTP to cyclic GMP (cGMP) in response to NO binding. NO-sGC-cGMP signalling influences an array of physiological processes including vascular tone, inflammation, fibrosis and metabolism.

sGC stimulators are small-molecule allosteric agonists that sensitise sGC to endogenous NO and increase cGMP production. They have shown activity in preclinical models of cardiovascular, renal, metabolic and fibrotic disorders [5-7]. These observations provide a rationale for clinical investigation of 
sGC stimulators for the treatment of diabetes and its complications, such as nephropathy, neuropathy and retinopathy.

Praliciguat (IW-1973), an investigational sGC stimulator, decreased BP and protected against end-organ damage in nonclinical disease models relevant to diabetes and hypertension [8]. In addition, it demonstrated positive metabolic effects in a diet-induced obesity mouse model [9] and reduced proteinuria and fasting blood glucose in the ZSF1 rat model of diabetic nephropathy [6]. In healthy adults, plasma cGMP increased and haemodynamic effects were observed when praliciguat 15-40 mg was administered daily for up to 21 days [10]. We conducted a Phase IIA exploratory trial to evaluate the safety, tolerability, pharmacokinetic and pharmacodynamic profile of praliciguat in individuals with type 2 diabetes and hypertension.

\section{Methods}

Study design and participants This was a randomised, double-blind, placebo-controlled, in-clinic, exploratory Phase IIA trial conducted at a single centre (ProSciento, Chula Vista, CA, USA). Eligible participants were aged 3075 years, had type 2 diabetes and hypertension for at least 6 months, and a BMI of $20-40 \mathrm{~kg} / \mathrm{m}^{2}$. They were required to be on a stable medication regimen for a minimum of 28 days prior to randomisation, including either an ACE inhibitor (ACEi) or an angiotensin receptor blocking agent (ARB), and at least one glucose-lowering agent. Additional BP- and glucose-lowering treatments were permitted. All participants were required to have baseline $\mathrm{HbA}_{1 \mathrm{c}} \leq 91 \mathrm{mmol} / \mathrm{mol}$ $(\leq 10.5 \%)$, fasting plasma glucose $\leq 13.3 \mathrm{mmol} / 1$, systolic BP (SBP) 120-160 mmHg and diastolic BP (DBP) 70$100 \mathrm{mmHg}$. Hepatic impairment, bleeding history/disorder, cancer, severe end-organ morbidity and the use of any investigational drug within 30 days were exclusionary. Pregnant and lactating women were excluded, and stringent birth control was required during the trial.

Three to twenty-eight days after a screening visit, 26 eligible participants were randomised in a 5:5:3 ratio to receive the following treatment: praliciguat $40 \mathrm{mg}$ once daily on days $1-$ $14(n=10)$; praliciguat $20 \mathrm{mg}$ twice daily on days $1-7$ then $40 \mathrm{mg}$ once daily on days 8-14 $(n=10)$; or placebo $(n=6)$. The computer-generated blocked randomisation schedule was prepared by an independent statistician, not otherwise associated with the study, using a block size of 13. Two praliciguat treatment regimens were tested to evaluate potential for different tolerability profiles. Praliciguat was provided as a $5 \mathrm{mg}$ oral tablet and placebo tablets matched praliciguat tablets in appearance. All participants were dosed with eight tablets (either praliciguat or placebo) twice daily throughout the 14 day treatment period to maintain the blind. Participants were discharged from the clinic on day 15 and returned for follow-up visits approximately 21 and 42 days after dose initiation.

The study was conducted in accordance with the Declaration of Helsinki and International Conference on Harmonisation (ICH) Guidelines for Good Clinical Practice. The study protocol, amendments and consent form were approved by the Institutional Review Board. All participants provided written informed consent before participation.

Pharmacokinetics Plasma concentrations of praliciguat were measured using a validated LC-tandem MS bioanalytical method, as previously described [10]. Non-compartmental pharmacokinetic calculations, using actual sampling times relative to dosing, were performed with Phoenix WinNonlin Version 6.4 (Certara LP, Princeton, NJ, USA) and included the maximum observed plasma concentration $\left(\mathrm{C}_{\max }\right)$, time of $\mathrm{C}_{\max }\left(\mathrm{T}_{\max }\right), \mathrm{AUC}$ for the plasma concentration-time curve, apparent volume of distribution during the terminal elimination phase $\left(\mathrm{V}_{\mathrm{z}} / \mathrm{F}\right)$ and the effective $t_{1 / 2}[11]$ (electronic supplementary material [ESM] Table 1).

Safety and pharmacodynamic assessments Treatment-emergent adverse events (TEAEs), vital signs, serum chemistry, haematology, coagulation and urinalysis (including urine creatinine) were assessed. Twenty-four-hour ambulatory BP monitoring (ABPM) with measurements at intervals of $30 \mathrm{~min}$ was conducted at baseline and on days 1, 7 and 14. To estimate insulin resistance, HOMA-IR was calculated from fasting plasma glucose and serum insulin levels [12]. The eGFR was calculated using the Chronic Kidney Disease Epidemiology Collaboration (CKD-EPI) creatinine equation [13]. Other assessments included reactive hyperaemia index (RHI) and augmentation index corrected to a heart rate of 75 beats/min (bpm) $\left(\mathrm{AI}_{75}\right)$, measured by digital plethysmography (EndoPAT, Itamar Medical, Caesarea, Israel). Inflammatory, vascular injury and lipoprotein biomarkers (ESM Table 2) were also assessed.

Platelet function was assessed by VerifyNow (both aspirin and P2 $\mathrm{Y}_{12}$ assays; Accumetrics, San Diego, CA, USA) and by flow cytometric measurement of both platelet surface activated glycoprotein IIb-IIIa and platelet surface P-selectin expression, as described previously [14]. These analyses were conducted with whole blood and platelet-rich plasma under both unstimulated and stimulated conditions with two platelet activators: ADP and thrombin receptor activating protein (TRAP) at two concentrations (Boston Children's Hospital Center for Platelet Research Studies).

Statistical analysis The planned sample size of 26 participants was determined outside of statistical considerations but was considered adequate to achieve the exploratory objectives of the trial. Data from all participants were used for all analyses. Analyses of change-from-baseline pharmacodynamic data 
Table 1 Participant characteristics at baseline

\begin{tabular}{|c|c|c|c|c|}
\hline \multirow[t]{2}{*}{ Characteristic } & \multirow{2}{*}{$\begin{array}{l}\text { Placebo } \\
(n=6)\end{array}$} & \multicolumn{3}{|l|}{ Praliciguat $40 \mathrm{mg}$ daily } \\
\hline & & $\begin{array}{l}\text { Twice daily / once daily } \\
(n=10)\end{array}$ & $\begin{array}{l}\text { Once daily / once daily }{ }^{\mathrm{b}} \\
(n=10)\end{array}$ & $\begin{array}{l}\text { Overall } \\
(n=20)\end{array}$ \\
\hline Age, years & $61 \pm 6$ & $61 \pm 8$ & $63 \pm 7$ & $62 \pm 7$ \\
\hline Male sex, $n(\%)$ & $2(33)$ & $6(60)$ & $5(50)$ & $11(55)$ \\
\hline \multicolumn{5}{|l|}{ Race/ethnicity, $n(\%)$} \\
\hline White & $6(100)$ & $7(70)$ & $7(70)$ & $14(70)$ \\
\hline Asian & 0 & $1(10)$ & $2(20)$ & $3(15)$ \\
\hline Black or African-American & 0 & $2(20)$ & $1(10)$ & $3(15)$ \\
\hline Hispanic or Latino & $5(83)$ & $2(20)$ & $3(30)$ & $5(25)$ \\
\hline Weight, kg & $87 \pm 20$ & $92 \pm 19$ & $82 \pm 20$ & $87 \pm 20$ \\
\hline BMI, $\mathrm{kg} / \mathrm{m}^{2}$ & $32 \pm 3$ & $33 \pm 4$ & $31 \pm 5$ & $32 \pm 5$ \\
\hline \multicolumn{5}{|l|}{ Haemodynamic variables } \\
\hline Systolic BP, mmHg & $132 \pm 8$ & $126 \pm 11$ & $129 \pm 6$ & $128 \pm 9$ \\
\hline Diastolic BP, mmHg & $77 \pm 9$ & $72 \pm 9$ & $72 \pm 6$ & $72 \pm 7$ \\
\hline MAP, mmHg & $96 \pm 8$ & $90 \pm 7$ & $91 \pm 6$ & $91 \pm 6$ \\
\hline Heart rate, bpm & $75 \pm 14$ & $73 \pm 11$ & $75 \pm 11$ & $74 \pm 11$ \\
\hline \multicolumn{5}{|l|}{ Metabolic variables } \\
\hline Plasma glucose, mmol/1 & $7.9 \pm 1.8$ & $9.0 \pm 2.8$ & $8.2 \pm 1.9$ & $8.6 \pm 2.4$ \\
\hline $\mathrm{HbA}_{1 \mathrm{c}}, \mathrm{mmol} / \mathrm{mol}$ & $60.1 \pm 14.3$ & $62.2 \pm 11.8$ & $63.7 \pm 11.8$ & $63.0 \pm 11.5$ \\
\hline $\mathrm{HbA}_{1 \mathrm{c}}, \%$ & $7.7 \pm 1.3$ & $7.8 \pm 1.1$ & $8.0 \pm 1.1$ & $7.9 \pm 1.1$ \\
\hline HOMA-IR ${ }^{c}$ & $7.4 \pm 5.2$ & $6.6 \pm 3.4$ & $7.2 \pm 5.7$ & $7.0 \pm .7$ \\
\hline Serum insulin, $\mathrm{pmol} / \mathrm{l}^{\mathrm{c}}$ & $146 \pm 107$ & $126 \pm 53$ & $123 \pm 91$ & $125 \pm 80$ \\
\hline Total cholesterol, mmol/1 & $4.0 \pm 1.3$ & $3.9 \pm 0.7$ & $4.3 \pm 0.9$ & $4.1 \pm 0.8$ \\
\hline LDL-cholesterol, mmol/1 & $1.9 \pm 1.1$ & $2.2 \pm 0.6$ & $2.1 \pm 0.9$ & $2.2 \pm 0.8$ \\
\hline HDL-cholesterol, mmol/1 & $1.2 \pm 0.3$ & $1.1 \pm 0.4$ & $1.2 \pm 0.3$ & $1.1 \pm 0.4$ \\
\hline Triacylglycerol, mmol/l & $1.7 \pm 0.7$ & $1.3 \pm 0.6$ & $2.3 \pm 1.4$ & $1.8 \pm 1.2$ \\
\hline 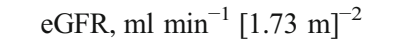 & $103 \pm 8$ & $89 \pm 20$ & $80 \pm 20$ & $85 \pm 20$ \\
\hline \multicolumn{5}{|l|}{ Medication, $n(\%)$} \\
\hline Metformin & $6(100)$ & $7(70)$ & $9(90)$ & $16(80)$ \\
\hline Sulfonylurea & $2(33)$ & $2(20)$ & $4(40)$ & $6(30)$ \\
\hline Dipeptidyl peptidase-4 inhibitor & $0(0)$ & $0(0)$ & $1(10)$ & $1(5)$ \\
\hline Insulin & $2(33)$ & $5(50)$ & $3(30)$ & $8(40)$ \\
\hline Statin & $3(50)$ & $7(70)$ & $8(80)$ & $15(75)$ \\
\hline ACEi & $2(33)$ & $7(70)$ & $6(60)$ & $13(65)$ \\
\hline $\mathrm{ARB}$ & $4(67)$ & $3(30)$ & $4(40)$ & $7(35)$ \\
\hline$\beta$-Adrenergic blocker & $1(17)$ & $1(10)$ & $1(10)$ & $2(10)$ \\
\hline Calcium channel blocker & $1(17)$ & $5(50)$ & $1(10)$ & $6(30)$ \\
\hline Diuretic & $1(17)$ & $3(30)$ & $3(30)$ & $6(30)$ \\
\hline Aspirin & $3(50)$ & $6(60)$ & $6(60)$ & $12(60)$ \\
\hline
\end{tabular}

Data are mean $\pm \mathrm{SD}$, unless otherwise indicated

a $20 \mathrm{mg}$ twice daily for 7 days, then $40 \mathrm{mg}$ once daily for 7 days

${ }^{\mathrm{b}} 40 \mathrm{mg}$ once daily for 14 days

${ }^{\mathrm{c}}$ Participants not on insulin ( $n=4$ for placebo group; $n=5$ for praliciguat twice daily/once daily group; $n=7$ for praliciguat once daily/once daily group)

were performed using ANCOVA with treatment as a fixed effect and baseline as a covariate. Least squares (LS) mean for each treatment and LS mean differences from placebo, along with the associated $95 \%$ CIs obtained from the model were rounded. Outcomes examined in this trial were not powered for inferential testing: analyses were descriptive and focused on estimation. All results should thus be considered exploratory and hypothesis-generating. All statistical 
analyses were performed using SAS Version 9.1 (SAS Institute, Cary, NC, USA).

\section{Results}

A total of 26 participants were randomised to one of the two praliciguat $40 \mathrm{mg}$ daily groups $(20 \mathrm{mg}$ twice daily for 7 days, then $40 \mathrm{mg}$ once daily for 7 days; $40 \mathrm{mg}$ once daily for 14 days; $n=10$ both groups) or the placebo group $(n=6)$. Of the 26 participants randomised, 25 (96\%) completed the study as planned. One person treated with praliciguat discontinued dosing due to an adverse event (described below). Baseline characteristics were generally similar, with no clinically significant differences, when comparing the praliciguat- and placebo-treated participants and the praliciguat twice daily, praliciguat once daily and placebotreated participants (Table 1). No participant changed their BP-lowering regimen during the treatment phase of the trial.

Praliciguat was rapidly absorbed, with a median $\mathrm{T}_{\max }$ of $1-$ $3 \mathrm{~h}$, and the two regimens produced similar dose-normalised $\mathrm{C}_{\max }$ (group mean: $5.84 \mathrm{ng} \mathrm{ml}^{-1} \mathrm{mg}^{-1}$ for twice daily dosing; $6.05 \mathrm{ng} \mathrm{ml}^{-1} \mathrm{mg}^{-1}$ for once daily dosing) after 7 days of dosing. After 14 days of dosing, the $\mathrm{V}_{\mathrm{z}} / \mathrm{F}$ was large (overall mean $4680 \mathrm{l}$ ), and the overall mean effective $t_{1 / 2}$ was $39.7 \mathrm{~h}$.
Detailed pharmacokinetic variables are presented in ESM Table 1.

Pharmacodynamic results after 14 days of treatment were similar for the two praliciguat regimens (twice daily/once daily vs once daily/once daily) and are presented combined. LS mean changes from baseline to day 14, as well as LS mean differences from placebo in metabolic variables, are presented in Fig. 1. Decreases from baseline in fasting plasma glucose were seen in both praliciguat and placebo groups, and the point estimate and associated 95\% CI for the difference suggests decreases were greater in praliciguat-treated patients (LS mean difference -0.7 [95\% CI $-1.8,0.4] \mathrm{mmol} / \mathrm{l}$ ). Changes from baseline in $\mathrm{HbA}_{1 \mathrm{c}}$ were small and similar in the praliciguat- and placebo-treated groups (LS mean [95\% $\mathrm{CI}]-0.3[-0.5,-0.2] \%(-3.6[-5.2,-2.0] \mathrm{mmol} / \mathrm{mol})$ and $-0.3[-0.6,-0.0] \%(-3.4[-6.3,-0.5 \mathrm{mmol} / \mathrm{mol}])$, respectively; LS mean difference from placebo $[95 \% \mathrm{CI}] 0[-0.3,0.3] \%$ $(-0.2[-3.5,3.2] \mathrm{mmol} / \mathrm{mol})$.

Among the subset of 16 participants taking oral glucoselowering agents but not insulin ( $n=12$ praliciguat, $n=4$ placebo), the point estimate and $95 \%$ CIs suggest the decrease from baseline in fasting plasma glucose was greater in participants treated with praliciguat than in those receiving placebo (LS mean $[95 \% \mathrm{CI}]-1.6[-2.1,-1.1] \mathrm{mmol} / \mathrm{l}$ and $-0.5[-1.3$, $0.3]$, respectively; LS mean difference from placebo [95\% CI] $-1.1[-2.0,-0.1] \mathrm{mmol} / \mathrm{l})$. In this subgroup, decreases
Fig. 1 Changes in metabolic variables from baseline to week 2 . Data are presented as LS mean change from baseline with $95 \%$ CIs. LS mean differences $(95 \%$ CI) between praliciguat $(n=19)$ and placebo-treated $(n=6)$ participants were as follows: (a) plasma glucose, $-0.7(-1.8,0.4)$ $\mathrm{mmol} / \mathrm{l} ;$ (b) total cholesterol, -0.7 $(-1.1,-0.2) \mathrm{mmol} / \mathrm{l}$; (c) LDLcholesterol, $-0.5(-1.0,-0.1)$ $\mathrm{mmol} / \mathrm{l}$; and (d) triacylglycerol, $-0.2(-0.5,0.2) \mathrm{mmol} / \mathrm{l}$ a

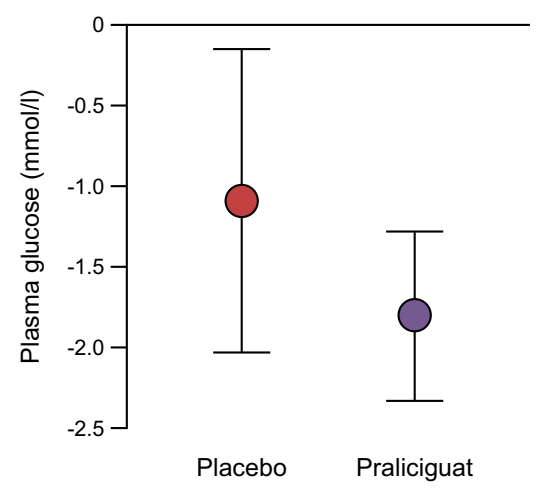

C

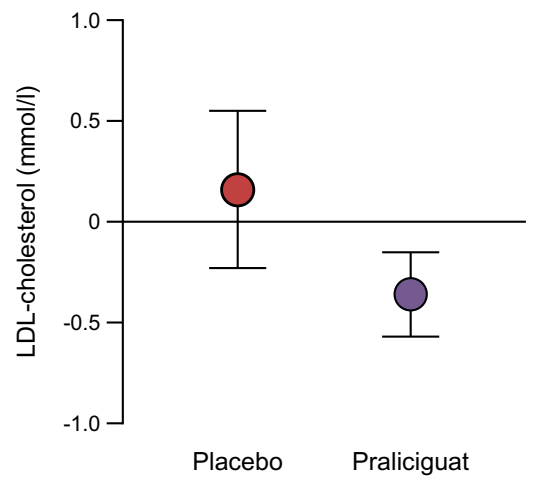

b

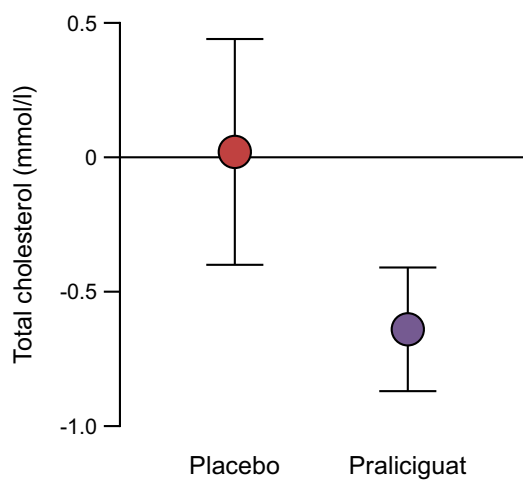

d

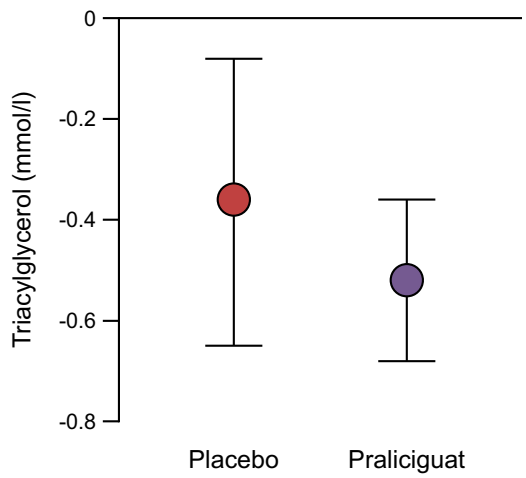


following praliciguat vs placebo treatment were also suggested for HOMA-IR (LS mean [95\% CI] $-36[-53,-19]$ and -13 $[-40,15]$, respectively; LS mean difference from placebo $[95 \%$ $\mathrm{CI}]-23[-56,9])$. In addition, LS mean change [95\% CI] from baseline in fasting serum insulin for praliciguat- and placebotreated participants was $-36[-54,-19] \mathrm{pmol} / \mathrm{l}$ and $-23[-54,7]$ $\mathrm{pmol} / \mathrm{l}$, respectively; LS mean difference from placebo $[95 \%$ CI $]-13[-49,22] \mathrm{pmol} / \mathrm{l}$.

Reductions from baseline in both total cholesterol and LDL-cholesterol were observed in praliciguat-treated participants (LS mean $[95 \% \mathrm{CI}]-0.6[-0.9,-0.4] \mathrm{mmol} / \mathrm{l}$ and -0.4 $[-0.6,-0.1] \mathrm{mmol} / \mathrm{l}$, respectively), while no reduction was observed in placebo-treated participants (LS mean $[95 \% \mathrm{CI}]$ $0.0[-0.4,0.4] \mathrm{mmol} / 1$ and $0.2[-0.2,0.5] \mathrm{mmol} / 1$, respectively). LS mean difference $(95 \% \mathrm{CI})$ from placebo for total cholesterol and LDL-cholesterol was $-0.7(-1.1,-0.2)$ $\mathrm{mmol} / \mathrm{l}$ and $-0.5(-1.0,-0.1) \mathrm{mmol} / 1$, respectively. Decreases from baseline in serum triacylglycerol were observed in both praliciguat- and placebo-treated participants, with the point estimate and 95\% CI suggesting greater reductions in those treated with praliciguat (LS mean difference $[95 \% \mathrm{CI}]-0.2[-0.5,0.2] \mathrm{mmol} / \mathrm{l})$. Changes from baseline in HDL-cholesterol levels were small and similar between praliciguat- and placebo-treated participants (LS mean [95\% CI $]-0.05[-0.14,0.03] \mathrm{mmol} / 1$ and $-0.01[-0.16,0.14]$ mmol/1, respectively; LS mean difference [95\% CI] for praliciguat vs placebo $-0.04[-0.21,0.13] \mathrm{mmol} / \mathrm{l})$. Point estimates and $95 \%$ CIs for changes from baseline in apolipoprotein B also suggested greater decreases in praliciguat-treated participants than in placebo-treated participants (LS mean difference $[95 \% \mathrm{CI}]-2.0 \times 10^{-4}\left[-6.0 \times 10^{-4}, 1.0 \times 10^{-4}\right]$ $\mathrm{mmol} / \mathrm{l}$; see ESM Table 2 for full results of apolipoprotein $\mathrm{B}$ and other lipoprotein biomarkers). Point estimates and $95 \%$ CIs for change from baseline in lipid levels for the subset of 18 participants on concomitant statin therapy (15 praliciguat, 3 placebo) also suggested greater declines in praliciguat-treated vs placebo-treated participants: total cholesterol (LS mean difference $[95 \% \mathrm{CI}]-0.4[-1.1,0.3] \mathrm{mmol} / \mathrm{l})$ and LDLcholesterol (LS mean difference $[95 \% \mathrm{CI}]-0.4[-1.1,0.2]$ $\mathrm{mmol} / \mathrm{l})$.

Neither body weight nor BMI changed from baseline over the treatment period for either praliciguat-treated or placebotreated participants, with no differences between the groups (LS mean difference [95\% CI] $0.0[-1.1,1.1] \mathrm{kg}$ and 0.0 $[-0.4,0.4] \mathrm{kg} / \mathrm{m}^{2}$, respectively).

Figure 2 presents changes from baseline in average $24 \mathrm{~h}$ haemodynamic variables as measured by ABPM. At day 14, point estimates and CIs suggest greater reductions from baseline for praliciguat-treated participants vs placebo-treated participants in average $24 \mathrm{~h}$ measurements (LS mean
Fig. 2 Changes in haemodynamic variables from baseline to week 2 measured by $24 \mathrm{~h}$ ABPM. Data are presented as LS mean change from baseline with $95 \%$ CIs. LS mean differences $(95 \% \mathrm{CI})$ between praliciguat-treated $(n=19)$ and placebo-treated $(n=6)$ participants were as follows: (a) systolic $\mathrm{BP},-2(-10,5) \mathrm{mmHg}$; (b) diastolic BP, $-4(-9,1)$ mmHg; (c) MAP, $-5(-10,1)$ $\mathrm{mmHg}$; and (d) heart rate, $3(-1$, 6) bpm a

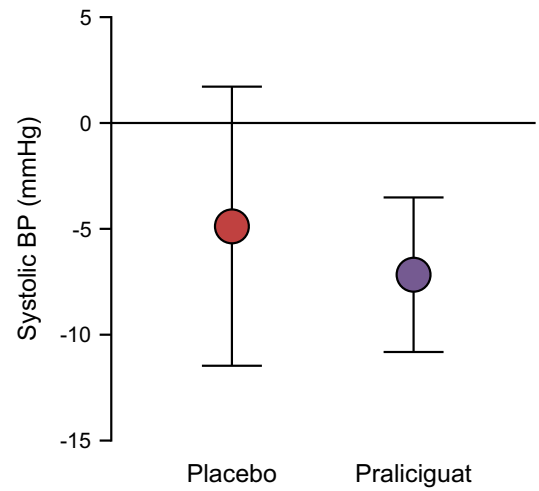

C

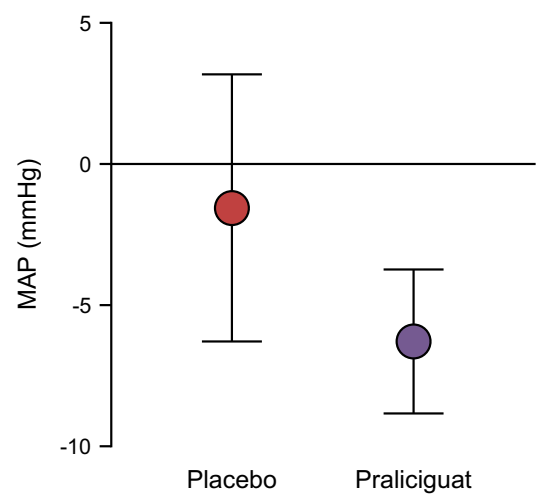

b

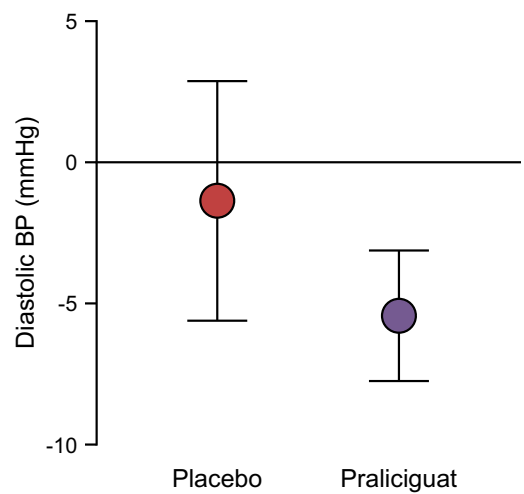

d

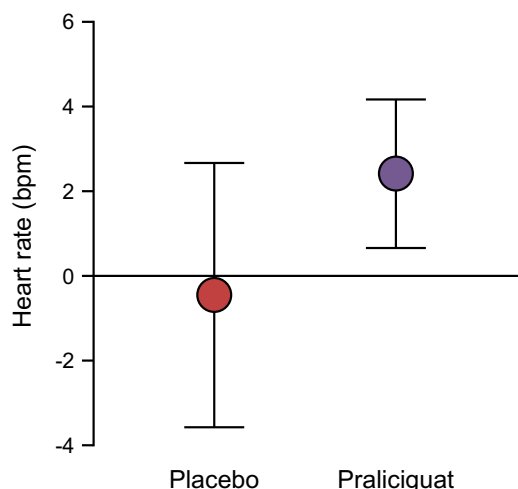


difference $[95 \% \mathrm{CI}]$ of $-2[-10,5] \mathrm{mmHg}$ for $\mathrm{SBP},-4[-9,1]$ $\mathrm{mmHg}$ for DBP and $-5[-10,1] \mathrm{mmHg}$ for mean arterial pressure [MAP]). Point estimates and 95\% CI for change in average $24 \mathrm{~h}$ heart rate suggested greater increases in praliciguat-treated participants than in placebo-treated participants (LS mean difference $3[95 \% \mathrm{CI}-1,6] \mathrm{bpm}$ ).

In post hoc analyses of subgroups stratified by median baseline BP, praliciguat-treated participants with baseline MAP $>92 \mathrm{mmHg}$ had a greater LS mean difference from placebo-treated participants $(-14[-23,-5] \mathrm{mmHg})$ than praliciguat-treated participants with baseline MAP $\leq 92 \mathrm{mmHg}$ (LS mean difference 2 [95\% CI $-4,8] \mathrm{mmHg}$ ). A similar pattern was seen for SBP and DBP.

At the pre-dose measurement on day 13, change from baseline in RHI was similar in praliciguat-treated and placebotreated participants (LS mean [95\% CI] $0.09[-0.17,0.34]$ and $0.28[-0.17,0.74]$, respectively; LS mean difference $[95 \% \mathrm{CI}]-0.20[-0.73,0.34]) . \mathrm{AI}_{75}$ declined from baseline in both praliciguat-treated and placebo-treated participants (LS mean $[95 \% \mathrm{CI}]-4.8[-9.0,-0.6]$ and $-0.99[-9.02$, 7.04], respectively; LS mean difference [95\% CI] -3.9 $[-13.2,5.5])$.

Changes from baseline and LS mean differences for biomarkers of inflammation and vascular injury are presented in ESM Table 2. Point estimates and associated 95\% CIs suggest that, compared with placebo-treated participants, praliciguat-treated participants had a greater decline in asymmetric dimethylarginine (ADMA; LS mean difference -10.7 $[95 \% \mathrm{CI}-18.7,-2.6] \mathrm{ng} / \mathrm{ml}$ ) and a greater increase in L-arginine/ADMA ratio (LS mean difference 22.2 [95\% CI 7.5, $36.8]$ ). Changes in other plasma biomarkers were similar between the two treatment groups.

There were no values or changes in values indicative of a praliciguat effect on platelet function as assessed by VerifyNow. This instrument measures platelet function using whole blood samples. For the VerifyNow $\mathrm{P}_{2} \mathrm{Y}_{12}$ assay, values below $180 \mathrm{P}_{2} \mathrm{Y}_{12}$ reaction units (PRU), a measure of ADPinduced platelet aggregation assessed by an increase in light transmission, suggest evidence of a $\mathrm{P}_{2} \mathrm{Y}_{12}$ inhibitor effect [15]. At both baseline and day 14, all participants had results above 180 PRU. For the VerifyNow aspirin test, values below 550 aspirin reaction units (ARU) indicate an aspirin-like inhibitory effect on arachidonic acid-induced platelet aggregation [16]. At baseline, all participants had values consistent with their reported aspirin use or no use, except for one person with reported concomitant aspirin use who had a value above 550 ARU. At day 14, three praliciguat-treated participants with reported concomitant aspirin use had values above 550 ARU, one praliciguat-treated participant with no report of concomitant aspirin use had a value below $550 \mathrm{ARU}$, and all other participants had values consistent with their reported aspirin use. In addition, praliciguat-treated participants did not show meaningful changes from baseline or differences from placebo in analyses of platelet surface activated glycoprotein IIb-IIIa and platelet surface P-selectin expression under unstimulated and stimulated conditions in both whole blood and platelet-rich plasma (data not shown).

Fourteen $(70 \%)$ of the 20 participants treated with praliciguat experienced at least one TEAE compared with five of six participants $(83 \%)$ treated with placebo (Table 2). One person taking praliciguat had a serious adverse event (SAE) and discontinued praliciguat after 11 days of treatment. All other participants completed 14 days of treatment.

All TEAEs other than the single SAE were characterised as mild. The most common TEAEs were headache $(5 / 20$ [25\%] praliciguat, $2 / 6$ [33\%] placebo), nausea $(5 / 20$ [25\%] praliciguat, 0/6 placebo) and hypoglycaemia (5/20 [25\%] praliciguat, 2/6 [33\%] placebo). Other TEAEs reported in two or more praliciguat-treated participants were diarrhoea (3/20 [15\%] praliciguat, 1/6 [17\%] placebo), abdominal pain $(2 / 20$ [10\%] praliciguat, 0 placebo $)$ and dyspepsia (2/20 [10\%] praliciguat, $0 / 6$ placebo).

Headache occurred in approximately the same proportion of praliciguat- and placebo-treated participants. Of the 3 of 10 (33\%) participants in the once daily/once daily praliciguat group who reported headache, all had onset in the first week of dosing. Of the 2 of 10 (20\%) participants in the twice daily/ once daily praliciguat group who reported headache, one had onset in the first week and one in the second week. Of the 2 of $6(33 \%)$ placebo-treated participants who reported headache, onset was reported by one in the first week and one in the second week.

Gastrointestinal TEAEs were reported in 9/20 (45\%) of praliciguat-treated participants, compared with $1 / 6(17 \%)$ of placebo-treated participants. Five of the praliciguat-treated participants reported multiple gastrointestinal events; all others reported a single event. Of the 5 of $10(50 \%)$ participants in the once daily/once daily praliciguat group who had at least one gastrointestinal adverse event, two had event onsets in both weeks, one had onset in the first week only, and two had onset in the second week. Of the 4 of 10 (40\%) participants in the twice daily/once daily praliciguat group, two had onsets in both weeks, one had onset only in the first week, and one only in the second week. The participant in the placebo group who experienced gastrointestinal adverse effects had onset in the second week.

All five praliciguat-treated participants who experienced hypoglycaemia were among the eight taking concomitant insulin, as was one of two placebo-treated participants who reported this adverse event.

Only two participants, one on the $40 \mathrm{mg}$ once daily dose of praliciguat and one on placebo, had an adverse event that could be consistent with low BP (dizziness).

A single SAE occurred in a 59-year-old man, who developed a spontaneous upper gastrointestinal haemorrhage after 11 days of dosing and was hospitalised for 1 day. Treatment 
Table 2 Treatment-emergent adverse events

\begin{tabular}{|c|c|c|c|c|}
\hline \multirow[t]{2}{*}{ MedDRA preferred term } & \multirow{2}{*}{$\begin{array}{l}\text { Placebo } \\
(n=6)\end{array}$} & \multicolumn{3}{|l|}{ Praliciguat $40 \mathrm{mg}$} \\
\hline & & $\begin{array}{l}\text { Twice daily / once daily } \\
(n=10)\end{array}$ & $\begin{array}{l}\text { Once daily / once daily }{ }^{\mathrm{b}} \\
(n=10)\end{array}$ & $\begin{array}{l}\text { Overall } \\
(n=20)\end{array}$ \\
\hline Any TEAE & $5(83)$ & $6(60)$ & $8(80)$ & $14(70)$ \\
\hline Headache & $2(33)$ & $2(20)$ & $3(30)$ & $5(25)$ \\
\hline Hypoglycaemia & $2(33)$ & $2(20)$ & $3(30)$ & $5(25)$ \\
\hline Nausea & 0 & $2(20)$ & $3(30)$ & $5(25)$ \\
\hline Diarrhoea & $1(17)$ & 0 & $3(30)$ & $3(15)$ \\
\hline Abdominal pain & 0 & 0 & $2(20)$ & $2(10)$ \\
\hline Dyspepsia & 0 & $1(10)$ & $1(10)$ & $2(10)$ \\
\hline Injection site haemorrhage & $3(50)$ & 0 & $1(10)$ & $1(5)$ \\
\hline Cough & 0 & 0 & $1(10)$ & $1(5)$ \\
\hline Dry throat & 0 & 0 & $1(10)$ & $1(5)$ \\
\hline Oropharyngeal pain & 0 & 0 & $1(10)$ & $1(5)$ \\
\hline Alopecia & 0 & 0 & $1(10)$ & $1(5)$ \\
\hline Gastrointestinal sounds abnormal & 0 & 0 & $1(10)$ & $1(5)$ \\
\hline Costochondritis & 0 & 0 & $1(10)$ & $1(5)$ \\
\hline Paronychia & 0 & 0 & $1(10)$ & $1(5)$ \\
\hline Dizziness & $1(17)$ & $1(10)$ & 0 & $1(5)$ \\
\hline Anaemia & 0 & $1(10)$ & 0 & $1(5)$ \\
\hline Eye irritation & 0 & $1(10)$ & 0 & $1(5)$ \\
\hline Dry mouth & 0 & $1(10)$ & 0 & $1(5)$ \\
\hline Eructation & 0 & $1(10)$ & 0 & $1(5)$ \\
\hline Gastroesophageal reflux disease & 0 & $1(10)$ & 0 & $1(5)$ \\
\hline Oesophagitis & 0 & $1(10)$ & 0 & $1(5)$ \\
\hline Upper gastrointestinal haemorrhage & 0 & $1(10)$ & 0 & $1(5)$ \\
\hline Vomiting & 0 & $1(10)$ & 0 & $1(5)$ \\
\hline Muscle spasms & 0 & $1(10)$ & 0 & $1(5)$ \\
\hline Pain in extremity & 0 & $1(10)$ & 0 & $1(5)$ \\
\hline Injection site injury & 0 & $1(10)$ & 0 & $1(5)$ \\
\hline Dermatitis contact & 0 & $1(10)$ & 0 & $1(5)$ \\
\hline Pseudohypoglycaemia & $1(17)$ & 0 & 0 & 0 \\
\hline Limb discomfort & $1(17)$ & 0 & 0 & 0 \\
\hline Tremor & $1(17)$ & 0 & 0 & 0 \\
\hline Nephrolithiasis & $1(17)$ & 0 & 0 & 0 \\
\hline Nocturia & $1(17)$ & 0 & 0 & 0 \\
\hline
\end{tabular}

Data are presented as $n(\%)$

a $20 \mathrm{mg}$ twice daily for 7 days, then $40 \mathrm{mg}$ once daily for 7 days

${ }^{\mathrm{b}} 40 \mathrm{mg}$ once daily for 14 days

MedDRA, Medical Dictionary for Regulatory Activities

was discontinued for the remainder of the trial. Prior to this episode, the participant's concomitant medications included captopril and insulin, and he had no history of gastrointestinal bleeding. Endoscopic evaluation identified a hiatal hernia with ulcerative oesophagitis but no source of active bleeding. The participant did not have further gastrointestinal bleeding and recovered uneventfully.

\section{Discussion}

This exploratory Phase IIA trial found that participants with type 2 diabetes and hypertension treated for 2 weeks with the sGC stimulator praliciguat had trends toward improvement in metabolic and haemodynamic outcomes. Decreases in fasting plasma glucose, insulin, HOMA-IR and serum lipids were seen in both praliciguat- and placebo-treated participants but 
the LS mean differences between the groups consistently suggested greater changes in praliciguat-treated participants. Glucose control as assessed by $\mathrm{HbA}_{1 \mathrm{c}}$ was unchanged, as expected in a trial of such short (14 days) treatment duration. Meaningful assessment of glucose control would need to be explored in longer clinical investigations.

Reduction in fasting plasma glucose in praliciguat-treated participants was suggested in those on stable regimens for glycaemic control, including the subset of participants who were not receiving concomitant insulin treatment. Praliciguatrelated decline in mean lipid levels was also observed in the subset of participants on concurrent statin therapy. These subgroup results suggest that praliciguat may provide additional glucose- and lipid-lowering effects on top of the current standard of care. However, these results should be interpreted with caution, as the subgroups were small.

Participants treated with praliciguat had a greater mean decrease from baseline in average $24 \mathrm{~h}$ BP compared with placebo. All participants in this trial were already on a stable antihypertensive regimen that included an ACEi or an ARB, suggesting an additional BP-lowering effect of praliciguat treatment. Reductions in BP are consistent with the known vasodilatory effects of modulators of the NO-sGC-cGMP signalling pathway [17-19]. The decreases in BP were seen predominantly in participants having baseline values above the median level for the cohort. Greater BP-lowering effects in participants with diabetes and inadequate BP control would be advantageous, especially if accompanied by few hypotension-associated adverse events, as in this trial.

Haemodynamic effects of sGC stimulation are known to be mediated through vascular smooth muscle but may be affected by endothelial NO release. In this trial, the effects of praliciguat on endothelial function were evaluated by peripheral arterial tonometry and by plasma biomarkers. Praliciguat showed no clear effect on RHI [20], a non-invasive tonometry measure of reactive changes in arterial tone. However, intraand inter-participant variability in RHI was high, and about half had baseline values corresponding to normal endothelial function. In contrast, praliciguat treatment was associated with reduction in plasma ADMA and increase in the L-arginine/ ADMA ratio. ADMA competitively inhibits the generation of NO from L-arginine by NO synthases. This, in turn, can reduce the bioavailability of $\mathrm{NO}$ and lead to endothelial dysfunction [21, 22]. Plasma ADMA is elevated in diabetes, hypertension and diabetic nephropathy, and is an independent risk factor for all-cause mortality and cardiovascular disease [23-25]. Thus, modulation of ADMA levels and L-arginine/ ADMA ratio by praliciguat may imply an impact on endothelial function which, if sustained, could be associated with cardiovascular and renal benefit.

The results from this trial support an extensive body of non-clinical evidence demonstrating that praliciguat and sGC stimulators lower BP, improve metabolic homeostasis by reducing blood glucose, increase insulin sensitivity, and reduce serum lipids $[7,26]$. Potential mechanisms for a positive metabolic effect of sGC stimulation could include promoting insulin access to tissues, enhancing insulin receptor signalling and/or improving mitochondrial function [26]. Non-clinical investigations have suggested an interdependence between insulin and the NO-sGC-cGMP signalling pathway in both the endothelium and metabolically active tissues that are disrupted in the metabolic syndrome [27, 28].

Reduction in total cholesterol, LDL-cholesterol and triacylglycerols could be mediated by enhanced insulin receptor signalling and/or improved function of proteins such as peroxisome proliferator-activated receptors (PPARs), key regulators of lipid and carbohydrate metabolism [29]. Dietary nitrates, which also increase plasma cGMP in humans [30], have been shown to increase metabolism of fatty acids in skeletal muscle through an $\mathrm{NO} / \mathrm{sGC} / \mathrm{cGMP} / \mathrm{PPAR}$-mediated mechanism in rats [29-31].

The pharmacokinetic profile of praliciguat in individuals with diabetes and hypertension was found to be consistent with that observed in healthy volunteers, including rapid absorption, a high volume of distribution suggesting extensive dispersal into tissues, and a $t_{1 / 2}$ supportive of once daily dosing [10]. The large volume of distribution is consistent with high tissue-to-plasma concentration ratios observed in animal studies of praliciguat $[8,9]$. High local drug concentrations can produce sustained pharmacological effects [32] and supports investigation in conditions associated with impaired tissue NO-sGC-cGMP signalling.

Overall, praliciguat was well tolerated in this study. Similar to reports from clinical trials of other sGC stimulators [33, 34] and consistent with the known pharmacological effects of drugs that modulate NO signalling, headache and gastrointestinal symptoms were among the most frequently observed TEAEs. These TEAEs were mild, transient and subsided with continued therapy. Headaches may be related to the BP-lowering effects of praliciguat; however, headaches in this trial occurred at a similar rate in placebo-treated participants. Among praliciguat-treated participants experiencing headache, most occurred in the first week in those who started on $40 \mathrm{mg}$ once daily dosing, suggesting both that this adverse event may be regimen-related and that 1 week of $20 \mathrm{mg}$ twice daily dosing may have reduced the likelihood of headache when the dose was changed to $40 \mathrm{mg}$ once daily in the second week. Gastrointestinal TEAEs, including nausea, diarrhoea, abdominal pain and dyspepsia, were the only types of event that were clearly imbalanced between praliciguat- and placebo-treated participants. $\mathrm{sGC}$ is present in smooth muscle cells, including in the gastrointestinal tract, and NO signalling plays a role in gastrointestinal physiological functioning, including gastrointestinal-tract motility [35, 36]. A similar pattern of gastrointestinal adverse effects is noted in the prescribing information for the only marketed sGC stimulator, riociguat (Adempas) [37]. 
Hypoglycaemia was reported with equal frequency in praliciguat- and placebo-treated participants, and, with the exception of a single placebo-treated individual, was observed exclusively in those receiving concomitant insulin therapy. This suggests that insulin and/or characteristics of individuals requiring insulin may have played a role in these events. Supporting this interpretation are results from another small trial of praliciguat in individuals with diabetes and hypertension, in which hypoglycaemia was reported in only one person on concomitant insulin, and other trials conducted in healthy volunteers where hypoglycaemia was not observed [10].

A single SAE occurred in this trial: an upper gastrointestinal haemorrhage event in a participant without a history of gastrointestinal bleeding. The prescribing information for riociguat contains a warning for bleeding because a disproportionate number of serious bleeding events were observed in Phase III trials [33, 34, 37] in individuals with pulmonary hypertension. Because sGC is expressed in platelets and NO-sGC-cGMP signalling has been reported to inhibit platelet activation [38], we assessed platelet function in this trial using multiple methods. Praliciguat treatment did not show an effect on platelet function, consistent with the results of prior clinical studies of praliciguat in healthy volunteers [10]. It is not known whether the large tablet burden required in this trial may have contributed to the SAE. A potential impact of praliciguat on platelet function and/or risk of serious bleeding events warrants continued clinical vigilance.

Several limitations of this study need to be noted. The trial was not powered for inference testing for any of the outcomes examined, so that all results must be considered hypothesisgenerating, requiring future confirmation. The trial examined only 26 individuals and the treatment duration of 14 days was too short to observe meaningful changes in long-term glycaemic control (e.g. $\mathrm{HbA}_{1 \mathrm{c}}$ levels) or to evaluate the durability of effects. The in-clinic treatment phase added lifestyle modification and imposed medication adherence as potential factors influencing the results. In addition, the number of placebo-treated participants was small, resulting in wide CIs for praliciguat-placebo treatment difference comparisons. Finally, trial entry criteria were liberal, allowing enrolment of individuals with type 2 diabetes and hypertension independent of treatment regimen.

The promising results observed in this study, including positive trends in metabolic and haemodynamic variables, support further clinical investigation of praliciguat. Praliciguat is being evaluated in separate Phase II trials in individuals with diabetic nephropathy and heart failure with preserved ejection fraction.

Acknowledgements The authors thank the participants in this study and acknowledge and thank P. Miller (Cyclerion Therapeutics) and the study teams (Cyclerion Therapeutics and ProSciento, Inc.) for their hard work conducting this study, and J. Jones (Cyclerion Therapeutics) for pharmacological insight. Some of the data were presented as abstracts at the ADA and EASD meetings in 2018.
Data availability The datasets generated during and/or analysed during the current study are not publicly available due to their proprietary nature but are available from the corresponding author on reasonable request.

Funding This trial was funded by Cyclerion Therapeutics. The funder was involved in the study design; collection, analysis and interpretation of the data; writing the report; and the decision to submit the report for publication.

Duality of interest LM is an employee of ProSciento, Inc., the contract research organisation that conducted the study. ALF and ADM are the principal investigators on a research grant to Boston Children's Hospital from Ironwood Pharmaceuticals. All other authors are employed by and may own stock/stock options in Cyclerion Therapeutics. No other potential conflicts of interest relevant to this article are reported.

Contribution statement All authors were involved in trial design and/or analysis and interpretation of the data. JPH and JPS drafted the manuscript; all other co-authors reviewed and revised it critically for important intellectual content. All authors had access to the data and gave final approval of the submitted version. JPH is the guarantor of this work and takes responsibility for the integrity of the data and the accuracy of the data analysis.

Open Access This article is licensed under a Creative Commons Attribution 4.0 International License, which permits use, sharing, adaptation, distribution and reproduction in any medium or format, as long as you give appropriate credit to the original author(s) and the source, provide a link to the Creative Commons licence, and indicate if changes were made. The images or other third party material in this article are included in the article's Creative Commons licence, unless indicated otherwise in a credit line to the material. If material is not included in the article's Creative Commons licence and your intended use is not permitted by statutory regulation or exceeds the permitted use, you will need to obtain permission directly from the copyright holder. To view a copy of this licence, visit http://creativecommons.org/licenses/by/4.0/.

\section{References}

1. National Center for Chronic Disease Prevention and Health Promotion, Division of Diabetes Translation (2014) National diabetes statistics report, 2014: estimates of diabetes and its burden in the United States. US Department of Health and Human Services Centers for Disease Control and Prevention, Atlanta

2. Tabit CE, Chung WB, Hamburg NM, Vita JA (2010) Endothelial dysfunction in diabetes mellitus: molecular mechanisms and clinical implications. Rev Endocr Metab Disord 11(1):61-74. https:// doi.org/10.1007/s11154-010-9134-4

3. Williams SB, Cusco JA, Roddy MA, Johnstone MT, Creager MA (1996) Impaired nitric oxide-mediated vasodilation in patients with non-insulin-dependent diabetes mellitus. J Am Coll Cardiol 27(3): 567-574. https://doi.org/10.1016/0735-1097(95)00522-6

4. van den Born JC, Hammes HP, Greffrath W, van Goor H, Hillebrands JL (2016) Gasotransmitters in vascular complications of diabetes. Diabetes 65(2):331-345. https://doi.org/10.2337/db151003

5. Sandner P (2018) From molecules to patients: exploring the therapeutic role of soluble guanylate cyclase stimulators. Biol Chem 399(7):679-690

6. Buys ES, Zimmer DP, Chickering J et al (2018) Discovery and development of next generation sGC stimulators with diverse 
multidimensional pharmacology and broad therapeutic potential. Nitric Oxide 78:72-80. https://doi.org/10.1016/j.niox.2018.05.009

7. Hoffmann LS, Etzrodt J, Willkomm L et al (2015) Stimulation of soluble guanylyl cyclase protects against obesity by recruiting brown adipose tissue. Nat Commun 6:7235

8. Tobin JV, Zimmer DP, Shea C et al (2018) Pharmacological characterization of IW-1973, a novel soluble guanylate cyclase stimulator with extensive tissue distribution, antihypertensive, antiinflammatory, and antifibrotic effects in preclinical models of disease. J Pharmacol Exp Ther 365(3):664-675. https://doi.org/ 10.1124/jpet.117.247429

9. Schwartzkopf CD, Hadcock J, Jones JE, Currie M, Milne GT, Masferrer J (2018) Praliciguat, a clinical-stage sGC stimulator, improved glucose tolerance and insulin sensitivity and lowered triglycerides in a mouse diet-induced obesity model. Diabetes 67(Suppl 1): 1886

10. Hanrahan JP, Wakefield JD, Wilson PJ et al (2019) A randomized, placebo-controlled, multiple-ascending-dose study to assess the safety, tolerability, pharmacokinetics, and pharmacodynamics of the soluble guanylate cyclase stimulator praliciguat in healthy subjects. Clin Pharmacol Drug Dev 8(5):564-575. https://doi.org/ 10.1002/cpdd. 627

11. Boxenbaum H, Battle M (1995) Effective half-life in clinical pharmacology. J Clin Pharmacol 35(8):763-766. https://doi.org/10. 1002/j.1552-4604.1995.tb04117.x

12. Matthews DR, Hosker JP, Rudenski AS, Naylor BA, Treacher DF, Turner RC (1985) Homeostasis model assessment: insulin resistance and beta-cell function from fasting plasma glucose and insulin concentrations in man. Diabetologia. 28(7):412-419. https://doi. org $/ 10.1007 / \mathrm{bf00280883}$

13. Levey AS, Stevens LA, Schmid CH et al (2009) A new equation to estimate glomerular filtration rate. Ann Intern Med 150(9):604 612. https://doi.org/10.7326/0003-4819-150-9-200905050-00006

14. Michelson AD (2004) Platelet function testing in cardiovascular diseases. Circulation 110(19):e489-e493. https://doi.org/10.1161/ 01.CIR.0000147228.29325.F9

15. Accriva Diagnostics. VerifyNow ${ }^{\circledR}$ PRUTest ${ }^{\circledR}$ platelet reactivity test: instructions for use. Available from: http://www.accriva.com/ uploads/literature/vn1016en-web-01.pdf. Accessed 14 Nov 2019

16. Accriva Diagnostics. VerifyNow ${ }^{\circledR}$ asprin platelet reactivity test: instructions for use. Available from: http:/www.accriva.com/ uploads/literature/vn1011en-web 00.pdf. Accessed 14 Nov 2019

17. Buys ES, Ko YC, Alt C et al (2013) Soluble guanylate cyclase $\alpha_{1^{-}}$ deficient mice: a novel murine model for primary open angle glaucoma. PLoS One 8(3):e60156. https://doi.org/10.1371/journal. pone. 0060156

18. Moncada S, Higgs EA (2006) Nitric oxide and the vascular endothelium. Handb Exp Pharmacol (176 Pt 1):213-254. https://doi.org/ 10.1007/3-540-32967-6 7

19. Moncada S, Palmer RM, Higgs EA (1988) The discovery of nitric oxide as the endogenous nitrovasodilator. Hypertension. 12(4): 365-372. https://doi.org/10.1161/01.hyp.12.4.365

20. Patvardhan EA, Heffernan KS, Ruan JM, Soffler MI, Karas RH, Kuvin JT (2010) Assessment of vascular endothelial function with peripheral arterial tonometry: information at your fingertips? Cardiol Rev 18(1):20-28. https://doi.org/10.1097/CRD. 0b013e3181c46a15

21. Sibal L, Agarwal SC, Home PD, Boger RH (2010) The role of asymmetric dimethylarginine (ADMA) in endothelial dysfunction and cardiovascular disease. Curr Cardiol Rev 6(2):82-90. https:// doi.org/10.2174/157340310791162659

22. Yu E, Ruiz-Canela M, Hu FB et al (2017) Plasma arginine/ asymmetric dimethylarginine ratio and incidence of cardiovascular events: a case-cohort study. J Clin Endocrinol Metab 102(6):18791888. https://doi.org/10.1210/jc.2016-3569

23. Triches CB, Mayer S, Quinto BMR, Batista MC, Zanella MT (2018) Association of endothelial dysfunction with cardiovascular risk factors and new-onset diabetes mellitus in patients with hypertension. J Clin Hypertens (Greenwich) 20(5):935-941

24. Schlesinger S, Sonntag SR, Lieb W, Maas R (2016) Asymmetric and symmetric dimethylarginine as risk markers for total mortality and cardiovascular outcomes: a systematic review and metaanalysis of prospective studies. PLoS One 11(11):e0165811. https://doi.org/10.1371/journal.pone.0165811

25. Schwedhelm E, Boger RH (2011) The role of asymmetric and symmetric dimethylarginines in renal disease. Nat Rev Nephrol 7(5):275-285. https://doi.org/10.1038/nrneph.2011.31

26. Pfeifer A, Kilic A, Hoffmann LS (2013) Regulation of metabolism by cGMP. Pharmacol Ther 140(1):81-91. https://doi.org/10.1016/j. pharmthera.2013.06.001

27. Cheng AM, Rizzo-DeLeon N, Wilson CL et al (2014) Vasodilatorstimulated phosphoprotein protects against vascular inflammation and insulin resistance. Am J Physiol Endocrinol Metab 307(7): E571-E579. https://doi.org/10.1152/ajpendo.00303.2014

28. Kang YM, Kim F, Lee WJ (2017) Role of NO/VASP signaling pathway against obesity-related inflammation and insulin resistance. Diabetes Metab J 41(2):89-95. https://doi.org/10.4093/dmj. 2017.41.2.89

29. Varga T, Czimmerer Z, Nagy L (2011) PPARs are a unique set of fatty acid regulated transcription factors controlling both lipid metabolism and inflammation. Biochim Biophys Acta 1812(8): 1007-1022. https://doi.org/10.1016/j.bbadis.2011.02.014

30. Kapil V, Milsom AB, Okorie M et al (2010) Inorganic nitrate supplementation lowers blood pressure in humans: role for nitritederived NO. Hypertension 56(2):274-281. https://doi.org/10.1161/ HYPERTENSIONAHA.110.153536

31. Ashmore T, Roberts LD, Morash AJ et al (2015) Nitrate enhances skeletal muscle fatty acid oxidation via a nitric oxide-cGMP-PPARmediated mechanism. BMC Biol 13:110

32. Xie X, Chen W, Zhang N et al (2017) Selective tissue distribution mediates tissue-dependent PPAR $\gamma$ activation and insulin sensitization by INT131, a selective PPAR $\gamma$ modulator. Front Pharmacol 8: 317

33. Ghofrani HA, D'Armini AM, Grimminger F et al (2013) Riociguat for the treatment of chronic thromboembolic pulmonary hypertension. N Engl J Med 369(4):319-329. https://doi.org/10.1056/ NEJMoa1209657

34. Ghofrani HA, Voswinckel R, Gall H et al (2013) Riociguat for pulmonary hypertension. Futur Cardiol 6(2):155-166

35. Groneberg D, Voussen B, Friebe A (2016) Integrative control of gastrointestinal motility by nitric oxide. Curr Med Chem 23(24): 2715-2735. https://doi.org/10.2174/0929867323666160812150907

36. Shah V, Lyford G, Gores G, Farrugia G (2004) Nitric oxide in gastrointestinal health and disease. Gastroenterology 126(3):903913. https://doi.org/10.1053/j.gastro.2003.11.046

37. Adempas [package insert] (2018) Bayer Healthcare Inc. Available from www.accessdata.fda.gov/drugsatfda_docs/label/2018/ 204819s011lbl.pdf. Accessed 15 Nov 2019

38. Smolenski A (2012) Novel roles of cAMP/cGMP-dependent signaling in platelets. J Thromb Haemost 10(2):167-176. https:// doi.org/10.1111/j.1538-7836.2011.04576.x

Publisher's note Springer Nature remains neutral with regard to jurisdictional claims in published maps and institutional affiliations. 